\title{
Spectra and time variability of Galactic black-hole X-ray sources in the low/hard state
}

\author{
D. Giannios ${ }^{1,2}$, N. D. Kylafis ${ }^{1,2}$, and D. Psaltis ${ }^{3}$ \\ ${ }^{1}$ University of Crete, Physics Department, PO Box 2208, 710 03, Heraklion, Crete, Greece \\ e-mail: dgiannios@physics.uoc.gr \\ ${ }^{2}$ Foundation for Research and Technology-Hellas, 711 10, Heraklion, Crete, Greece \\ ${ }^{3}$ University of Arizona, 1118 E. 4th St., Tucson, AZ 85721, USA
}

Received 1 April 2004 / Accepted 24 May 2004

\begin{abstract}
We propose a jet model for the low/hard state of galactic black-hole X-ray sources which explains a) the X-ray spectra; b) the time-lag spectra; c) the increase of the variability amplitude (QPO and high frequency) with increasing photon energy; and d) the narrowing of the autocorrelation function with increasing photon energy. The model (in its simplest form) assumes that i) there is a uniform magnetic field along the axis of the jet; ii) the electron density in the jet is inversely proportional to distance; and iii) the jet is "hotter" near its center than at its periphery. We have performed Monte Carlo simulations of Compton upscattering of soft photons from the accretion disk and have found power-law high-energy spectra with photon-number index in the range 1.5-2, power-law time lags versus Fourier frequency with index $\sim 0.8$, and an increase of the rms amplitude of variability and a narrowing of the autocorrelation function with photon energy as has been observed in Cygnus X-1. Similar energy spectra and time variability have been observed in neutron-star systems in the island state. It is therefore quite likely that a similar model holds for these sources as well.
\end{abstract}

Key words. accretion, accretion disks - black hole physics - radiation mechanisms: non-thermal - methods: statistical X-rays: stars

\section{Introduction}

Galactic black-hole binaries have been extensively observed for more than thirty years. Their spectral and timing behavior exhibits a great variety of properties, yet in most cases they can be classified to be in one of few different states. The X-ray spectra of black-hole candidates have two distinct components: a multi-color black-body component (Mitsuda et al. 1984; but see Merloni et al. 2000), widely accepted to come from the accretion disk (Shakura \& Sunyaev 1973), and a non-thermal hard X-ray tail that is usually assumed to come from unsaturated inverse Compton scattering of soft photons with energetic electrons (see e.g. Sunyaev \& Titarchuk 1980; Poutanen 2001). The relative strength of the thermal component in comparison with the hard tail, the slope of the tail, and the variability are used to identify the spectral state of the source at a given epoch. When the luminosity of the source is low (less than a few percent of the Eddington luminosity) and the thermal component is weak or absent, with the non-thermal tail dominant and the rms variability of the source high $(20 \%-50 \%)$, then the source is in the low/hard state (see e.g. van der Klis 1995).

The location of the energetic electrons that Comptonize a soft photon input (and result in the power-law emission) is model-dependent. One possibility is that hot, optically thin plasma exists in a corona (Galeev et al. 1979; Haardt \& Maraschi 1993; Haardt et al. 1994; Stern et al. 1995; Poutanen \& Fabian 1999) that lies above and below the thin disk. A second possibility is a two-temperature flow (Shapiro et al. 1976; Ichimaru 1977; Rees et al. 1982; Narayan \& Yi 1994; Abramowicz et al. 1995; Esin et al. 1998).

There is growing evidence that all X-ray binaries harboring a black hole display radio emission when they are in the low/hard X-ray state (Hjellming \& Han 1995; Fender et al. 1997; Mirabel et al. 1998; Fender 2001; Stirling et al. 2001; Gallo et al. 2003). Inverse Compton scattering by relativistic electrons in a jet has been proposed as a mechanism for the production of X-rays and $\gamma$-rays in Active Galactic Nuclei (Begelman \& Sikora 1987; Bednarek et al. 1996; Harris \& Krawczynski 2002) and X-ray binaries (Band \& Grindlay 1986; Levinson \& Blandford 1996; Georganopoulos et al. 2002; Romero et al. 2002). There might also be a significant contribution of the synchrotron emission from the jet to the hard X-rays (Markoff et al. 2001; Corbel \& Fender 2002).

Spectral information alone, however, cannot reveal the accretion geometry. This is because rather different models can result in very similar spectra. To make further progress in our understanding of how accretion works, studies of the timing properties of these sources are also essential. 
The fact that phase lags between two different X-ray bands are approximately independent of Fourier frequency (Miyamoto et al. 1988) is impossible to explain with models where the Comptonization is taking place in a hot, uniform, optically thin plasma. This led to the idea of a large hot Comptonizing medium with density inversely proportional to radius (Hua et al. 1997; Kazanas et al. 1997; Kazanas \& Hua 1999; Hua et al. 1999). Still this model failed to explain that the autocorrelation function of the light curve of Cygnus X-1 in the low/hard state becomes narrower as the photon energy increases (Maccarone et al. 2000).

Recently, Reig et al. (2003) proposed that inverse Compton scattering of soft disk photons by energetic electrons in a jet can explain both the X-ray emission of black-hole candidates in the low/hard state and the decreasing time lags with Fourier frequency. The density of the electrons in this (quite simple) jet model is assumed to drop inversely proportional to the vertical distance $z$ from the black hole. In such a case, and for an effective optical depth to electron scattering of order unity along the axis of the jet, there is equal probability for scattering of the photons per decade of distance $z$. The smallest period of variability that can pass through the scattering medium is of the order of the timelag caused by scattering, which is of the order of $z / c$. Faster variability gets smoothed out. This means that the maximum frequency of variability that can pass through the jet is inversely proportional to the timelag. Thus, timelags roughly inversely proportional to Fourier frequency can be produced. Applying this model to Cygnus X-1 it was possible to reproduce both the energy and the time-lag spectra.

In this work we make one simple modification to the model described by Reig et al. (2003). Namely, we assume that the electrons are more energetic at small polar distances $\rho$ (in the core of the jet) than at larger $\rho$ (periphery of the jet). With this modification we are able to explain two additional observational facts exhibited by Cygnus $\mathrm{X}-1$. One is the narrowing of the autocorrelation function with increasing photon energy (Maccarone et al. 2000) and the other is the hardening of the high-frequency power spectrum with increasing photon energy (Nowak et al. 1999). Preliminary results of this work appeared in Kylafis et al. (2004). A similar approach was followed by Lehr et al. (2000) to explain the energy dependence of the fractional amplitude of the high-frequency QPOs in GRS $1915+105$.

\section{The model}

\subsection{Characteristics of the jet}

Our jet model is quite similar to the one described in Reig et al. (2003). More specifically, we assume that the jet is accelerated close to its launching area and maintains a constant (mildly relativistic) velocity thereafter. Furthermore, and for computational simplicity, we assume that a uniform magnetic field exists along the axis of the jet (taken to be the $z$ axis). The electron velocity has two components; one parallel $\left(v_{\|}\right)$and one perpendicular $\left(v_{\perp}\right)$ to the magnetic field.

The new ingredient of the model is that the perpendicular component of the velocity is not constant, but drops linearly with polar distance $\rho$, with its value in the outer edge of the jet being a fraction (typically half) of that along the $z$ axis. The result is a jet whose core is "hotter" than its periphery. This, rather reasonable, assumption will have important implications for the shapes of the autocorrelations and the power-density spectra of the light curves in different energy bands.

The electron-density profile in the jet is taken to be of the form

$n_{\mathrm{e}}(z)=n_{0}\left(z_{0} / z\right)^{p}$,

where $z$ is the vertical distance from the black hole and $p$ is a parameter close to 1 (see Sect. 3 below). The parameters $n_{0}$ and $z_{0}$ are the electron density and the height at the base of the jet respectively. If $H$ is the extent of the jet along the $z$ axis, then for $p=1$ the Thomson optical depth along the axis of the jet is

$\tau_{\|}=n_{0} \sigma_{T} z_{0} \ln \left(H / z_{0}\right)$.

Let $\pi R^{2}(z)$ be the cross-sectional area of the jet as a function of height $z$. Then from the continuity equation $\dot{M}=$ $\pi R^{2}(z) m_{\mathrm{p}} n_{\mathrm{e}}(z) v_{\|}$we obtain the radial extent $R$ of the jet as a function of height $z$

$R(z)=\left(R_{0}^{2} z / z_{0}\right)^{1 / 2}$

Here $R_{0}=\left(\dot{M} / \pi m_{\mathrm{p}} n_{0} v_{\|}\right)^{1 / 2}$ is the radius of the jet at its base, $\dot{M}$ is the mass ejection rate, and $m_{\mathrm{p}}$ is the proton mass.

\subsection{The Monte Carlo simulations}

For our Monte Carlo simulations we follow Cashwell \& Everett (1959) and Pozdnyakov et al. (1983). A code similar to ours, albeit independent, was used in the work of Reig et al. (2001, 2003). The two codes are in excellent agreement. The procedure we follow is described briefly below.

Photons from the accretion disk, in the form of a blackbody distribution of characteristic temperature $T_{\mathrm{bb}}$, are injected at the base of the jet with an upward isotropic distribution. It is not clear how the blackbody temperature of the disk varies with polar distance $\rho$, since we do not have a pure accretion disk, but we also have ejection of matter from the disk in the form of a jet. Thus, for simplicity, we consider a constant $T_{\mathrm{bb}}$ and constant luminosity per unit polar distance $\rho$. These assumptions are not crucial for our conclusions. We also consider disk photons that are emitted at poloidal distances $\rho<R_{0}$. This is justified by the fact that, for the typical values of $R_{0}$ considered here $\left(\sim 10^{8} \mathrm{~cm}\right.$, see Sect. 3$)$, the disk flux outside this radius is small and, furthermore, only a small fraction of it will interact with the jet.

Each photon is given a weight equal to unity when it leaves the accretion disk, and its time of flight is set equal to zero. The optical depth $\tau(\hat{n})$ along the photon's direction $\hat{n}$ is computed and a fraction $\mathrm{e}^{-\tau(\hat{n})}$ of the photon's weight escapes and is recorded. The rest of the weight of the photon is scattered in the jet. If the effective optical depth in the jet is significant (i.e., $\gtrsim 1$ ), then a progressively smaller and smaller weight of the photon experiences more and more scatterings. When the 
remaining weight in a photon becomes less than a small number (typically $10^{-8}$ ), we start with a new photon.

The time of flight of a random walking photon (or more accurately of its remaining weight) is updated at every scattering by adding the last distance traveled divided by the speed of light. For the escaping weight along a travel direction we add an extra time of flight outside the jet in order to bring in step all the photons (or fractions of them) that escape in a given direction from different points of the "surface" of the jet. The longer a fractional photon stays in the jet, the more energy it gains on average from the circular motion (i.e., $\boldsymbol{v}_{\perp}$ ) of the electrons. Such Comptonization can occur everywhere in the jet. Yet, a photon that random-walks throughout the jet has a longer time of flight than a photon that random walks near the bottom of the jet.

If the defining parameters of a photon (position, direction, energy, weight, and time of flight) at each stage of its flight are computed, then we can determine the spectrum of the radiation emerging from the scattering medium and the time delay of each escaping fractional photon. The optical depth to electron scattering, the energy shift, and the new direction of the photons after scattering are computed using the corresponding relativistic expressions.

\subsection{The high-frequency power spectrum}

The power spectra of Cygnus X-1 in the low/hard state can be well fitted by a doubly broken power low (Churazov et al. 2001; see, however, Nowak 2000; Belloni et al. 2002; Pottschmidt et al. 2003, for a different approach; and Revnivtsev et al. 2000 for evidence of a break above $\sim 40 \mathrm{~Hz}$ ). Other black-hole candidates show similar power spectra when they are in the low/hard state (Miyamoto et al. 1992). The first break is typically observed at frequencies of the order of $0.1 \mathrm{~Hz}$, where the slope changes from zero to $\sim-1$ (when the power is expressed in units of $\mathrm{rms}^{2} / \mathrm{Hz}$ ) and the second break at about $1 \mathrm{~Hz}$ with the high Fourier-frequency slope being -1.5.

Nowak et al. (1999) showed, for Cygnus X-1, that while the slopes of the first two power laws (slopes $\sim 0$ and $\sim-1$ ) do not depend on photon energy, the high-frequency power law exhibits a characteristic hardening with increasing photon energy. In this work we show that this hardening can be explained by a jet that has a core "hotter" than its periphery. Note, however, that other black-hole candidates show an energy-independent power-density spectral shape (Lin et al. 1999). In the context of our model, this means that the jets in these sources have $v_{\perp}(\rho) \approx$ constant.

In order to compute the power spectrum of the light curves in different energy bands, further assumptions concerning the source of the X-ray variability have to be made. We assume that the variability originates in the accretion disk, which is the source of the soft-photon input. This variability is modified as the disk photons travel through the jet and create the power-law energy spectrum.

We think that it is reasonable to assume that the highest frequency of variability, produced in a ring of disk material at radius $\rho$, is of the order of the Keplerian frequency at this radius, namely

$\nu_{\mathrm{K}}(\rho)=1 /(2 \pi)\left(G M / \rho^{3}\right)^{1 / 2}$

where $M$ is the mass of the black hole. This assumption is justified by the small number of characteristic frequencies that describe the power spectra (Belloni et al. 2002). This implies that a Fourier frequency $v$ is associated with variability in the part of the accretion disk with radius

$\rho<(2 \pi v)^{-2 / 3}(G M)^{1 / 3}$

On the other hand, we have assumed that the core of the jet is "hotter", resulting in a harder energy spectrum for input soft photons that come from the region close to the black hole. This is because these photons sample the core of the jet more than its periphery. Thus, higher Fourier frequencies are naturally associated with harder energy spectra, making the power spectra flatter in the harder energy bands. This is exactly what is observed in Cygnus X-1 (Nowak et al. 1999) and it will be quantitatively presented in Sect. 4.3. Furthermore, in the context of this model, the break of the power spectrum that occurs at a frequency of a few $\mathrm{Hz}$ is naturally attributed to the Keplerian frequency at the outer edge $\rho=R_{0}$ of the base of the jet.

\section{Parameter values}

Our jet model has a number of free parameters. The values of these parameters are chosen so that they are reasonable, and they produce results similar to the ones observed.

The parameters of our model are the following: The exponent $p$ (Eq. (1)), the temperature $T_{\mathrm{bb}}$ of the soft photon input, the extent $H$ of the jet, the radius $R_{0}$ and the height $z_{0}$ of the base of the jet, the Thomson optical depth $\tau_{\|}$along the axis of the jet, the parallel component of the velocity $v_{\|}$of the electrons, the perpendicular velocity component $v_{\perp, 0}$ at $\rho=0$, and its value at the outer edge $(\rho=R)$ of the jet $v_{\perp, \text { out }}$. We assume that $v_{\perp}$ drops linearly with radius $\rho$, but any other smooth drop gives qualitatively similar results. We remark here that, due to the mildly relativistic flow in the jet, the emerging X-ray spectra from the jet depend on the angle between the line of sight and the magnetic field direction.

The values of the parameters that reproduce quite well the observations of Cygnus X-1 are here called reference values and they are: $p=1, k T_{\mathrm{bb}}=0.2 \mathrm{keV}, H=10^{5} r_{\mathrm{g}}, R_{0}=100 r_{\mathrm{g}}$, $z_{0}=5 r_{\mathrm{g}}, \tau_{\|}=2.5, v_{\|}=0.8 \mathrm{c}$, and $v_{\perp, 0}=0.55 \mathrm{c}=2 v_{\perp, \mathrm{out}}$, where $r_{\mathrm{g}}=G M / c^{2}=1.5 \times 10^{6} \mathrm{~cm}$ stands for the gravitational radius of a 10 solar-mass black hole.

To have good statistics in our Monte Carlo results, we combined all the escaping photons with directional cosine with respect to the axis of the jet in the range $0.2<\cos \theta<0.6$. Strictly speaking, this is inconsistent with our assumption of a uniform magnetic field. If the magnetic field were uniform, then only one value of $\cos \theta$ would be appropriate for each source. As we will see (Sect. 4), a range of directional cosines is absolutely necessary to reproduce the observed phenomena. Therefore, a non-uniform (probably a spiraling) magnetic field permeates the jet. 


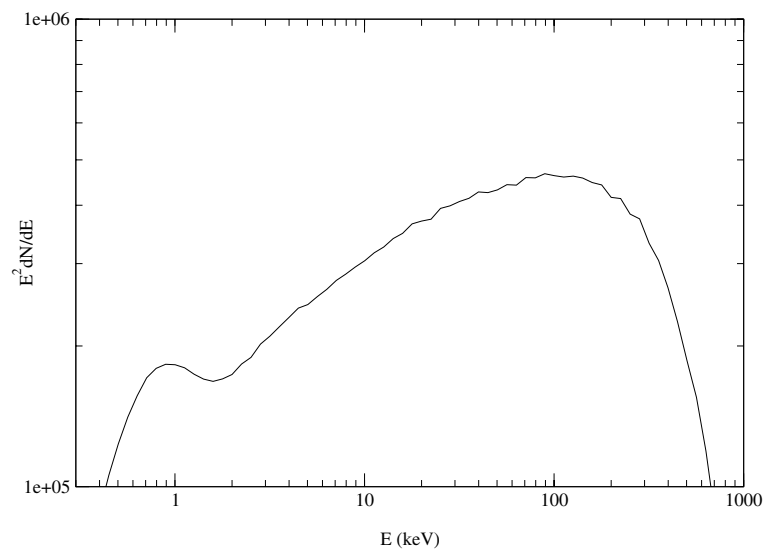

Fig. 1. Emergent photon-number spectrum multiplied by $E^{2}$ from our jet model for the reference values of the parameters given in Sect. 3. The soft X-ray excess below $1 \mathrm{keV}$ is essentially the unscattered softphoton input. When fitted with a power law with an exponential cutoff (see Eq. (6)), we find $\Gamma=1.7$ and $E_{\text {cut }}=280 \mathrm{keV}$.

Besides the reference values, we have also explored what range of the parameter space is compatible with the observations. Fortunately, this range is rather large. To quantify the last statement, we have found that a variation by a factor of two of parameters such as $H, \tau_{\|}, R_{0}, z_{0}$ and/or by $10 \%$ of the velocity components of the electrons or $p$ does not alter our conclusions.

\section{Results and discussion}

In what follows, we present results for the energy and time-lag spectra, the autocorrelation and cross correlation functions, and the power-density spectra.

\subsection{Energy and time-lag spectra}

The energy spectrum of the emerging photons for the reference values of the parameters (previous section) is shown in Fig. 1. The spectrum above $\sim 2 \mathrm{keV}$ can be well fitted with a power law with an exponential cutoff

$\mathrm{d} N / \mathrm{d} E \propto E^{-\Gamma} \mathrm{e}^{-E / E_{\text {cut }}}$,

where $\Gamma=1.7$ and $E_{\text {cut }}=280 \mathrm{keV}$. One can also see the soft $\mathrm{X}$-ray spectrum (below $\sim 2 \mathrm{keV}$ ), which is essentially the unscattered part of the soft-photon input.

Keeping the rest of the parameters at their reference values and varying the Thomson optical depth along the axis of the jet $\tau_{\|}$, we explore the role of this parameter on the emerging energy spectrum (Fig. 2). As expected, higher optical depths result in harder spectra (the photons are scattered more and gain more energy from the energetic electrons of the jet). The photon number spectral index $\Gamma$ decreases from 2 to 1.45 , when $\tau_{\|}$ varies in the range $1.5-4$.

We also made a similar study of the role of the radius $R_{0}$ of the base of the jet. By increasing $R_{0}$, the spectrum hardens and vice versa (Fig. 3). This is so because a larger $R_{0}$ translates to a larger Thomson optical depth perpendicular to the jet axis (we kept the rest of the parameters at their reference values) and therefore more photon scatterings.

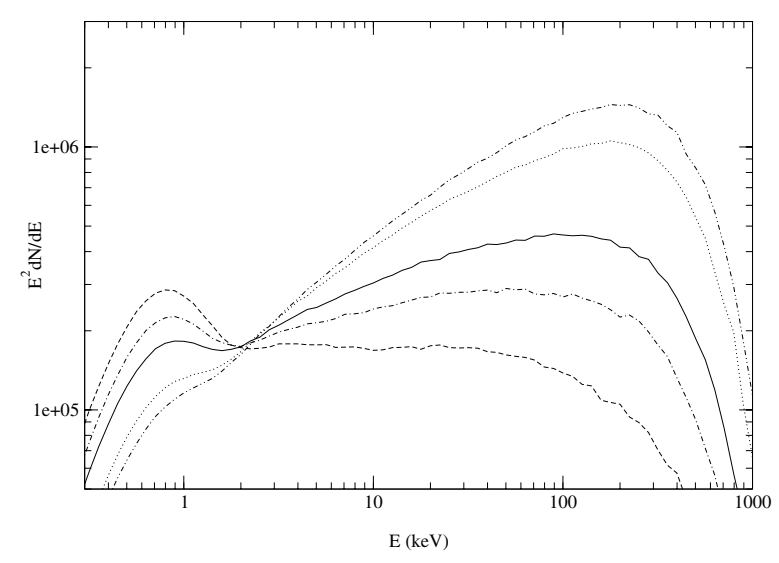

Fig. 2. Emergent photon-number spectra multiplied by $E^{2}$ from our jet model for different optical depths $\tau_{\|}$. The dashed, dash-dotted, solid, dotted and dash-double-dotted curves correspond to $\tau_{\|}=1.5,2,2.5$, 3.5 , and 4 respectively. The rest of the parameters are kept at their reference values. The power-law slope decreases with increasing optical depth from $\Gamma \simeq 2$ (for $\tau_{\|}=1.5$ ) to $\Gamma \simeq 1.45$ (for $\tau_{\|}=4$ ). The cutoff energy $E_{\text {cut }}$ varies slowly in the region $260-300 \mathrm{keV}$. For all the curves, the same number of input photons $N=4 \times 10^{6}$ was used.

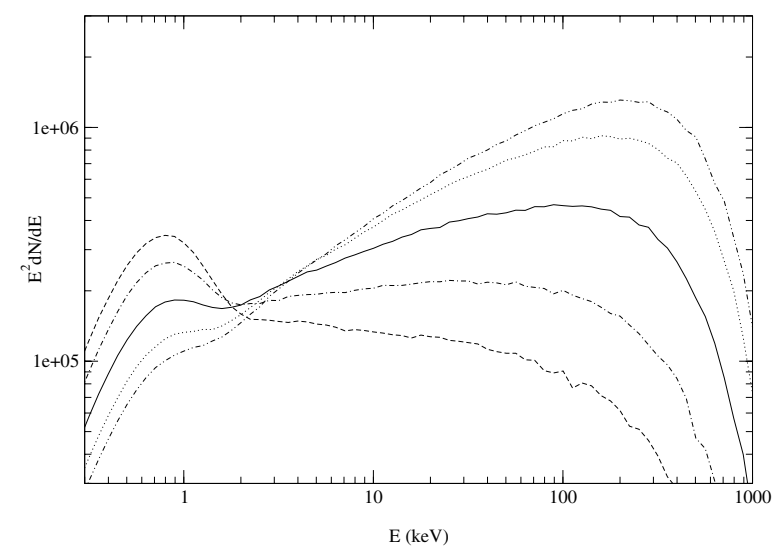

Fig. 3. Emergent photon-number spectra multiplied by $E^{2}$ from our jet model for different radii of the base of the jet. Here the dashed, dash-dotted, solid, dotted, and dash-double-dotted curves correspond to $R_{0} / r_{\mathrm{g}}=30,50,100,200$, and 300 respectively. The rest of the parameters are kept at their reference values. The spectra harden as $R_{0}$ increases, because the optical depth to electron scattering increases along the direction perpendicular to the axis of the jet. For all the curves, the same number of input photons $N=4 \times 10^{6}$ was used.

The cutoff energy $E_{\text {cut }}$ has a weak dependence on $\tau_{\|}$and $R_{0}$, but a rather strong dependence on $v_{\perp}$. This is because $v_{\perp}$ determines the maximum energy gain of the soft photons.

We then turn our attention to the timing properties that can be extracted from our model and compare them with the observations. We start with the frequency-dependent time lags between two different energy bands. To simulate them, the time of flight of all escaping photons is recorded in 16384 time bins, each of $1 / 256 \mathrm{~s}$ duration. The time of flight is computed by adding up the path lengths traveled by each photon and dividing the sum by the speed of light. Then we consider the light curves of two energy bands, $0.2-4 \mathrm{keV}$ and $14-45 \mathrm{keV}$, to compare our results with observations directly (e.g. Nowak et al. 1999). Following Vaughan \& Nowak (1997), we compute the 


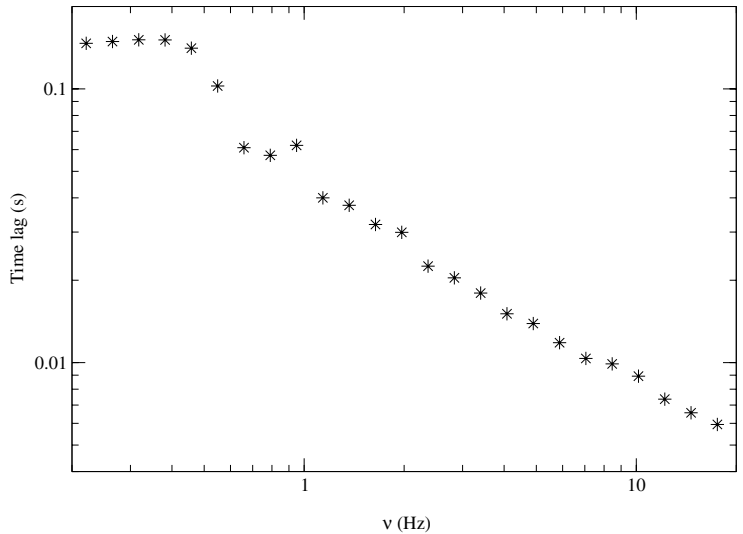

Fig. 4. Computed time lags as a function of Fourier frequency when the reference values of the parameters are used. Positive lags indicate that hard photons lag soft photons. The results are logarithmically rebinned for clarity. The best-fit power law (in the $0.2-20 \mathrm{~Hz}$ frequency range) is $v^{-0.8}$.

phase lags and through them the time lags between the two energy bands as a function of Fourier frequency.

Figure 4 shows the time lags as a function of Fourier frequency for the fixed values of the parameters (Sect. 3). The time-lag spectrum is approximately represented by a power law $v^{-\beta}$, with index $\beta=0.8$ in the frequency range $0.2-20 \mathrm{~Hz}$, in good agreement with the observations of Cyg X-1 and GX 339-4 (Nowak et al. 1999; Ford et al. 1999). The lowfrequency $(\sim 0.4 \mathrm{~Hz})$ break of the time lags is determined by the range of directional cosines with respect to the magnetic field direction in the jet (Sect. 3). A larger range would produce a break at lower Fourier frequencies and vice versa.

The time lags depend (among other things) on the dimensions of the jet. The scale of the jet is set by $R_{0}$ and $z_{0}$ (see Eq. (3)). To quantify this dependence, we consider a "large" jet $\left(R_{0}=300 r_{\mathrm{g}}, z_{0}=20 r_{\mathrm{g}}\right)$ and a scaled down one $\left(R_{0}=50 r_{\mathrm{g}}\right.$, $z_{0}=2 r_{\mathrm{g}}$ ). In Fig. 5 the time lags are presented for these two parameter sets and it is obvious that the larger jet results in larger lags by a factor of 2 for the same frequency. Notice, however, that the frequency dependence of the lags is very similar.

Pottschmidt et al. (2000) studied the evolution of the lags for different epochs of Cygnus X-1 and noticed that the lags have a tendency to increase in magnitude during state transitions. In the context of our model, this can be understood as the association of the state transitions with a more extended jet.

\subsection{Auto- and cross-correlation functions}

Maccarone et al. (2000) computed the autocorrelation functions (ACF) of the light curves of Cygnus X-1 in different energy bands and showed that the width of the ACF decreases with photon energy. This imposes strong constraints on the different models that attempt to describe both the spectral and the temporal properties of accreting black-hole binaries.

With the simulated light curves of our jet model at hand (for the reference values of the parameters given in Sect. 3), it is straightforward to calculate the ACF at two different energy bands viz. 0.2-4 keV (soft band) and 14-45 keV (hard band). The ACFs are normalized to unity at zero lag in both

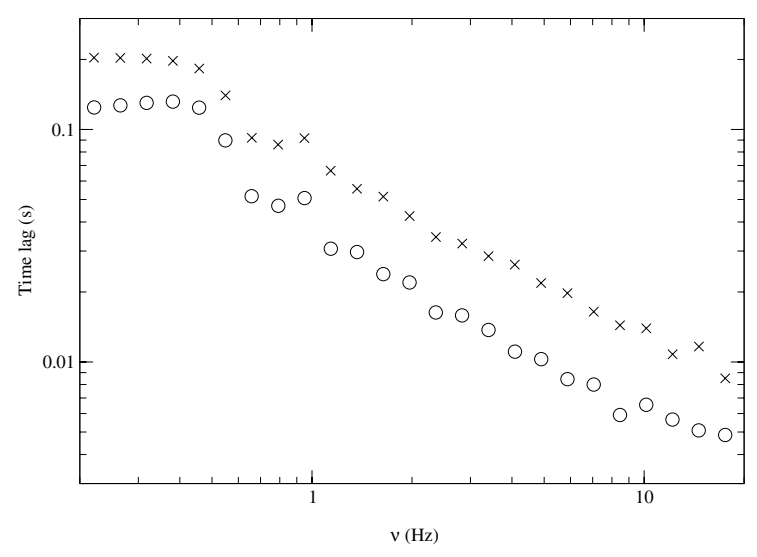

Fig. 5. Computed time lags as a function of Fourier frequency for different dimensions of the jet. The crosses correspond to a large jet with $R_{0}=300 r_{\mathrm{g}}$ and $z_{0}=20 r_{\mathrm{g}}$, and the open circles to a smaller jet with $R_{0}=50 r_{\mathrm{g}}$ and $z_{0}=2 r_{\mathrm{g}}$. The lags of the bigger jet are a factor of 2 larger than those of the smaller one at the same frequency. In either case, the best-fit power law (in the $0.2-20 \mathrm{~Hz}$ frequency range) is $v^{-0.8}$.

bands and are shown in Fig. 6. We find that the width of the ACF decreases with photon energy, in qualitative agreement with the observations. At first sight, this is counter-intuitive. It can never happen in a spherical Comptonizing medium. However, in our model the high-energy photons are not escaping isotropically, i.e., with a uniform distribution in $\cos \theta$. The more energetic photons come out preferentially at large values of $\cos \theta$ and with a lightcurve which is narrower than that of the less energetic photons. This gives rise to a narrowing of the ACF with increasing photon energy. In a realistic model with a non-uniform magnetic field, this could come about as follows: High-energy photons (escaping with large values of $\cos \theta$ ) come from relatively small regions of the jet and have a narrower lightcurve than the softer photons which come from the entire jet.

In Fig. 6 we also plot the cross-correlation function of the two light curves. It should be stressed that the exact shape of the auto- and cross-correlation functions depends on the properties of the shots of the soft-photon input.

\subsection{Power-density spectra}

Following the method described in Sect. 2.3, we calculate the high-frequency part of the power spectrum in five different photon energy bands (0.2-4 keV, 4-6.3 keV, 6.3-9 keV, 9-14 keV, and $14-45 \mathrm{keV})$.

In the context of our model, the second break in the power spectrum corresponds to the Keplerian frequency at $\rho=R_{0}$ in the accretion disk, which for $R_{0}=100 r_{\mathrm{g}}=1.5 \times 10^{8} \mathrm{~cm}$ is $v_{\mathrm{br}} \simeq 3 \mathrm{~Hz}$. At higher frequencies, the rms soft-input variability is assumed to have a power-law frequency dependence

$\mathrm{rms}_{\text {in }}=\mathrm{rms}_{0}\left(\frac{v}{3 \mathrm{~Hz}}\right)^{-1 / 3}$,

so as to match the high-frequency slope of the power spectrum for low-energy photons $(E \sim 1 \mathrm{keV})$. Note that for $\mathrm{rms}_{\text {in }} \propto$ $v^{-1 / 3}$ we have $\mathrm{rms}_{\text {in }}^{2} / \mathrm{Hz} \propto v^{-5 / 3}$. Here $\mathrm{rms}_{0}$ is the observed rms at $3 \mathrm{~Hz}$. 


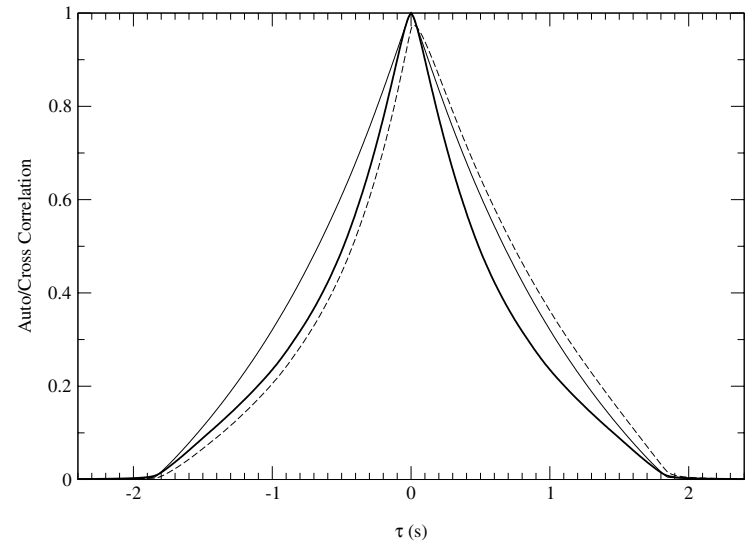

Fig. 6. The autocorrelation functions of the simulated light curves in two energy bands. The thin solid curve corresponds to the soft band $(0.2-4 \mathrm{keV})$, and the thick solid curve to the hard band (14-45 keV). Both curves are normalized to 1 for lag $\tau=0$. Notice that the autocorrelation function becomes narrower in the higher energy band. We also plot (dashed curve) the cross-correlation function of the two light curves.

We remind the reader that, according to our assumptions (Sect. 2.3), the variability at a given Fourier frequency $v$ is identified with the one that comes from the part of the accretion disk with $r$ constrained by expression (5). Thus, for a given Fourier frequency $v$, we vary the soft-photon input from the appropriate part of the accretion disk by an amount determined by Eq. (7) and record the resulting variability in the different energy bands. We find that the higher the Fourier frequency the larger the fractional change in $\mathrm{rms}^{2} / \mathrm{Hz}$ between the soft band $(0.2-4 \mathrm{keV})$ and one of the higher bands (Fig. 7). This can be understood as follows: The higher the Fourier frequency the closer to the core of the jet the source of variability is and thus the soft-input photons get upscattered in a "hotter" medium. In the construction of our model, the curves of $\mathrm{rms}^{2} / \mathrm{Hz}$ for the different energy bands meet at $\sim v_{\mathrm{br}}$, which corresponds to $r=R_{0}$, i.e., the entire accretion disk considered in our model.

As a consequence of the above, the high-frequency $(v>$ $v_{\text {br }}$ ) slope of the power spectrum depends on the energy band. A power-law fit to the power spectrum for the soft energy band $(0.2-4 \mathrm{keV})$ results in an index 1.7, essentially unchanged from the input value 5/3. As the band energy increases, the power spectrum flattens and for the hardest energy band considered (14-45 keV) the index becomes 1.4 (Fig. 8), in agreement with the observations of Cyg X-1 (Nowak et al. 1999). This variation of the index by $\sim 0.3$ is a consequence of the negative radial gradient of the perpendicular component of the electron velocity $v_{\perp}$ and depends on how much more energetic are the electrons in the core of the jet in comparison to those at its periphery. Here we have taken $v_{\perp, 0}=0.55 c=2 v_{\perp, \text { out }}$. Had we taken $v_{\perp, 0}=v_{\perp, \text { out }}$ (as in Reig et al. 2003), no flattening of the power-density spectra with photon energy would have been produced. This may be the case for some black-hole candidates (Lin et al. 1999).

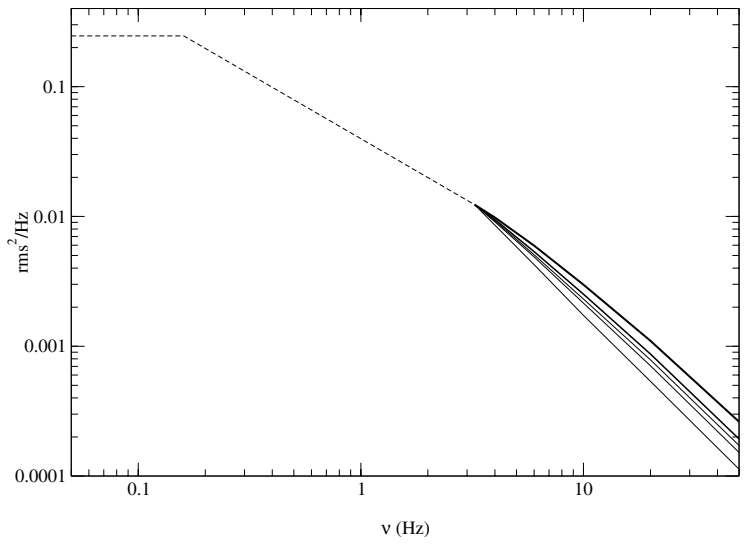

Fig. 7. Power-density spectrum of Cyg X-1. The dashed line corresponds to the low-frequency part of the power spectrum, as typically observed. The part of the power spectrum above the second break is the result of our simulations and shows the dependence of the spectrum on the photon energy. The five curves correspond to different energy bands as follows: $b_{1}:(0.2-4), b_{2}:(4-6.3), b_{3}:(6.3-9), b_{4}$ : (9-14), $b_{5}$ : (14-45) keV. The steepest slope (thinnest curve) corresponds to $b_{1}$, the second in steepness to $b_{2}$ and so forth.

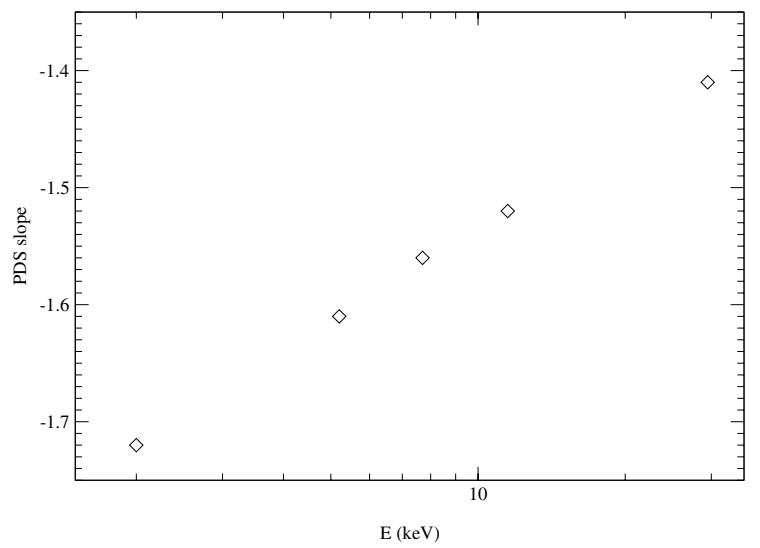

Fig. 8. Power-law slope of the high-frequency power-density spectra (Fig. 7) versus photon energy. The abolute value of the slope for the different energy bands is $b_{1}: 1.72, b_{2}: 1.61, b_{3}: 1.56, b_{4}: 1.52, b_{5}: 1.41$.

\section{Conclusion}

The non-thermal X-ray emission in black-hole binaries is usually assumed to come from inverse Compton scattering of soft photons in a hot corona (e.g. Poutanen \& Fabian 1999) that lies above and below a thin disk (Shakura \& Sunyaev 1973), or in a two-temperature flow (Shapiro et al. 1976). Here we explore an alternative picture where the inverse Compton scattering takes place in the jet whose presence, whenever the source is in the low/hard state, is well established (Fender 2001; Stirling et al. 2001; Gallo et al. 2003).

In a previous work (Reig et al. 2003) it was shown that, for a range of the parameters of a very simple jet model, both the energy spectra and the frequency-dependent time lags of Cygnus X-1 could be reproduced. In this work we have added a negative gradient along the polar direction $r$ of the electron velocity component $v_{\perp}$, resulting in a jet whose core is "hotter" than its periphery. This jet model can reproduce not only the energy spectrum and the time lags but also the fact that the 
autocorrelation function is measured to be narrower (Macarrone et al. 2000) and the high-frequency power spectrum harder (Nowak et al. 1999) for the harder photon energy bands.

In the context of this model the X-ray variability comes from the soft photon input that is further reprocessed in the jet. The high frequency break in the power spectrum (typically seen at $\sim 1 \mathrm{~Hz}$ ) is identified with the Keplerian frequency at the outer edge of the base of the jet.

One important challenge to our model is to demonstrate that, with no change of the assumptions, one can explain quantitatively the observed radio spectrum of black-hole sources in the low/hard state (Fender 2001) and the correlation between radio and X-ray luminosities (Gallo et al. 2003). Preliminary calculations indicate that this is possible. A detailed presentation is upcoming (Giannios, in preparation).

Neutron-star systems in the island state exhibit energy spectra and time variability similar to those of black-hole systems. We therefore suggest that our model may be relevant for these neutron-star systems as well.

Acknowledgements. One of us (NDK) would like to thank Iosif Papadakis for useful discussions. This research has been supported in part by the Program "Heraklitos" of the Ministry of Education of Greece.

\section{References}

Abramowicz, M. A., Chen, X. M., Kato, S., Lasota, J. P., \& Regev, O. 1995, ApJ, 438, L37

Band, D., \& Grindlay, J. E. 1986, ApJ, 311, 595

Bednarek, W., Kirk, J. G., \& Mastichiadis, A. 1996, A\&AS, 120, 571

Begelman, M. C., \& Sikora, M. 1987, ApJ, 322, 650

Belloni, T., Psaltis, D., \& van der Klis, M. 2002, ApJ, 572, 392

Cashwell, E. D., \& Everett, C. J. 1959, A Practical Manual on the Monte Carlo Method for Random Walk Problems (Oxford: Pergamon)

Churazov, E., Gilfanov, M., \& Revnivtsev, M. 2001, MNRAS, 321, 759

Corbel, S., \& Fender, R. P. 2002, ApJ, 573, 35

Esin, A. A., McClintock, J. E., \& Narayan, R. 1998, ApJ, 500, 523

Fender, R. P. 2001, MNRAS, 322, 31

Fender, R. P.. Pooley, G. G., Brocksopp C., \& Newell, S. J. 1997, MNRAS, 290, L65

Ford, E. C., van der Klis, M., Méndez, M., van Paradijs, J., \& Kaaret, P. 1999, ApJ, 512, L31

Galeev, A. A., Rosner, R., \& Vaiana, G. S. 1979, ApJ, 229, 318

Gallo, E., Fender, R. P., \& Pooley, G. G. 2003, MNRAS, 344, 60

Georganopoulos, M., Aharonian, F. A., \& Kirk, J. G. 2002, A\&A, 388, L25

Haardt, F., \& Maraschi, L. 1993, ApJ, 413, 507
Haardt, F., Maraschi L., \& Ghisellini, G. 1994, ApJ, 432, L95

Harris, D. E., \& Krawczynski, H. 2002, ApJ, 565, 244

Hjellming, R. M., \& Han, X. 1995, in Radio properties of X-ray binaries, ed. W. H. G. Lewin, J. van Paradijs, \& E. P. J. van der Heuvel, $\mathrm{X}$-ray binaries (Cambridge: Cambridge University Press), 308

Hua, X., Kazanas, D., \& Titarchuk, L. 1997, ApJ, 482, L57

Hua, X., Kazanas, D., \& Cui, W. 1999, ApJ, 512, 793

Ichimaru, S. 1977, ApJ, 214, 840

Kazanas, D., Hua, X., \& Titarchuk, L. 1997, ApJ, 480, 735

Kazanas, D., \& Hua, X. 1999, ApJ, 519, 750

Kylafis, N. D., Giannios, D., \& Psaltis, D. 2004, in X-ray Timing 2003: Rossi and Beyond, ed. P. Kaaret, F. K. Lamb, \& J. H. Swank, in press

Lehr, D. E., Wagoner, R. V., \& Wilms, J. 2000 [arXiv:astro-ph/0004211]

Levinson, A., \& Blandford, R. 1996, ApJ, 456, L29

Lin, D., Smith, I. A., Böttcher, M., \& Liang, E. P. 1999, ApJ, 531, 963 Maccarone, T. J., Coppi, P. S., \& Poutanen, J. 2000, ApJ, 537, L107

Markoff, S., Falcke, H., \& Fender, R. 2001, A\&A, 372, L25

Merloni, A., Fabian, A., \& Ross, R. 2000, MNRAS, 313, 193

Mirabel, I. F., Dhawan, V., Chaty, S., et al. 1998, A\&A, 330, L9

Mitsuda, K., Inoue, H., Koyama, K., et al. 1984, PASJ, 36, 741

Miyamoto, S., Kitamoto, S., Mitsuda, K., \& Dotani, T. 1988, Nature, 336,450

Miyamoto, S., Kitamoto, S., Iga, S., Negoro, H., \& Terada, K. 1992, ApJ, 391, L21

Narayan, R., \& Yi, I. 1994, ApJ, 428, L13

Nowak, M. A. 2000, MNRAS, 318, 361

Nowak, M. A., Vaughan, B. A., Wilms, J., Dove, J. B., \& Begelman, M. C. 1999 , ApJ, 510, 874

Pottschmidt, K., Wilms, J., Nowak, M. A., et al. 2000, A\&A, 357, L17

Pottschmidt, K., Wilms, J., Nowak, M. A., et al. 2003, A\&A, 407, 1039

Poutanen, J. 2001, Adv. Space Res., 28, 267

Poutanen, J., \& Fabian, A. C. 1999, MNRAS, 306, L31

Pozdnyakov, L. A., Sobol, I. M., \& Sunyaev, R. A. 1983, Astrophys. \& Space Phys. Rev., 2, 189

Rees, M. J., Phinney, E. S., Begelman, M. C., \& Blandford, R. P. 1982, Nature, 295, 17

Reig, P., Kylafis, N. D., \& Spruit, H. C. 2001, A\&A, 375, 155

Reig, P., Kylafis, N. D., \& Giannios, D. 2003, A\&A, 403, L15

Revnivtsev, M., Gilfanov, M., \& Churazov, E. 2000, A\&A, 363, 1013

Romero, G. E., Kaufman Bernadó, M. M., \& Mirabel, F. 2002, A\&A, 393, L61

Stern, B. E., Poutanen, J., Svensson, R., Sikora, M., \& Begelman, M. C. 1995, ApJ, 449, L13

Stirling, A. M., Spencer, R. E., de la Force, C. J., et al. 2001, MNRAS, 327,1273

Shakura, N. I., \& Sunyaev, R. A. 1973, A\&A, 24, 337

Shapiro, S. L., Lightman, A. P., \& Eardley, D. M. 1976, ApJ, 204, 187

Sunyaev, R. A., \& Titarchuk, L. G. 1980, A\&A, 86, 121

van der Klis, M. 1995, in The lives of neutron stars, ed. M. A. Alpar, Ü. Kiziloglu, \& J. van Paradijs (Kluwer Academic Publishers), NATO ASI Ser., C450

Vaughan, B. A., \& Nowak, M. A. 1997, ApJ, 474, L43 